\title{
ON THE CHOW-FORMS OF ELLIPTIC NORMAL CURVES OF DEGREE 4
}

\author{
By
}

\author{
Tatsuji TANAKA
}

\begin{abstract}
In this note, we shall aim, using explicit theta constants, to describe the Chow-form of an elliptic normal curve of degree 4 embedded in $\boldsymbol{P}^{3}$ by using theta functions, at any point of the upper half plane. In this case, the embedded elliptic curve is a complete intersection which is defined by two quadratic forms. In order to calculate its Chow-form, we use the elimination theory. Our main result is Th. $1^{\prime}(\S 2)$. In our case, Chow-forms are divisors of the Grassmann variety of lines in $\boldsymbol{P}^{3}$. From the theorem, we see that the Chow-forms of elliptic normal curves of degree 4 lie on a linear subspace $M$ (cf. 2.3) of dimension $23 . \S 2$ is the main part of this note.
\end{abstract}

In $\S 3$, using the theory of elliptic modular forms of level 4 , we consider the geometric meaning of Th. 1'. Then our theorem shows that the Chow point is given by modular forms of weight 2 (of weight 1 in the traditional sense) and of level 4 . The compactification of the moduli space of level 4 is isomorphic to a plane conic. Thus, we see that the Chow points of the projective elliptic normal curves of degree 4 determined by the points of the upper half plane form a rational curve of degree 4 in $M$ which is essentially the image of the 2-uple embedding of the above plane conic (Th. 2, §3. cf. Comments after the proof of Th. 2). Furthermore, we see that the correspondence which assigns the Chow point to a point of the upper half plane can be extended to the inequivalent cusps of the principal congruence subgroup $\Gamma(4)$ of level 4 (Cor. 1, $\S 3$ ). Finally, we give a remark about the structure of the corresponding component of the Chow variety parametrizing 1-cycles of degree 4 in $P^{3}$ (Cor. 2, §3). 


\section{§1. Situations}

This $\S$ is the part of grounds on which this note is founded.

First, we prepare some notations. $\boldsymbol{Z}, \boldsymbol{Q}, \boldsymbol{R}$ and $\boldsymbol{C}$ are as usual the ring of rational integers, the field of rational numbers, field of real numbers and the field of complex numbers, respectively.

$H$ is the upper half plane of $\boldsymbol{C}$, i.e., $\boldsymbol{H}=\{\tau \in \boldsymbol{C} \mid \operatorname{Im}(\tau)>0\}$.

We put $\boldsymbol{e}(t)=\exp (2 \pi \sqrt{-1} t)$ in general. For $k=\left(k_{1} k_{2}\right)$ with $k_{1}$ and $k_{2} \in \boldsymbol{R}$, we define a holomorphic function $\vartheta_{k}(\tau \mid z)$ on $H \times C$ by

$$
\vartheta_{k}(\tau \mid z)=\sum_{r \in \boldsymbol{Z}} \boldsymbol{e}\left[\frac{1}{2}\left(k_{1}+r\right) \tau\left(k_{1}+r\right)+\left(k_{1}+r\right)\left(z+k_{2}\right)\right] \quad((\tau, z) \in \boldsymbol{H} \times \boldsymbol{C}) .
$$

For any $\tau \in H$, we denote by $\Lambda_{\tau}, E_{\tau}$ a lattice subgroup $\boldsymbol{Z}+\boldsymbol{Z} \tau$ of $\boldsymbol{C}$, a complex torus $C / \Lambda_{\tau}$.

Let $\tau$ be an arbitrary point of $H$. For simplicity, for $i, j \in Z$, we put $\vartheta_{i j}(\tau \mid z)=$ $\vartheta_{i / 2 j / 2}(\tau \mid z)$. When $\tau$ is fixed, we denote by $\vartheta_{i j}(0)$ the theta constants $\vartheta_{i j}(\tau \mid 0)$. If $(i, j) \neq(1,1)$, for any $\tau \in H, \vartheta_{i j}(\tau \mid 0) \neq 0$. Moreover, we have Jacobi's identity

$$
\vartheta_{00}(0)^{4}=\vartheta_{01}(0)^{4}+\vartheta_{10}(0)^{4} .
$$

We define a holomorphic embedding $\phi: E_{\tau} \rightarrow \boldsymbol{P}^{3}$ by

$$
\forall z \in C ; \quad \phi(z)=\left(\vartheta_{00}(\tau \mid 2 z), \vartheta_{01}(\tau \mid 2 z), \vartheta_{10}(\tau \mid 2 z), \vartheta_{11}(\tau \mid 2 z)\right) .
$$

Since $\left\{\vartheta_{i / 4} 0(\tau \mid z) \mid 0 \leq i \leq 3\right\}$ is linearly independent and determines the same very ample line bundle as $\left\{\vartheta_{i j}(\tau \mid 2 z) \mid i, j=0,1\right\}$ (e.g. using $(\theta 5)$ in [5], p. 50), the embedding $\phi$ is the usual embedding of the torus $E_{\tau}([6])$ followed by a projective transformation.

Let $X_{\tau}$ be the image curve $\phi\left(E_{\tau}\right) \subset \boldsymbol{P}^{3}$, and let $\left(x_{0}, x_{1}, x_{2}, x_{3}\right)$ be the homogeneous coordinates in $\boldsymbol{P}^{3}$ corresponding to the above map.

The curve $X_{\tau}$ is non-singular of degree 4 and defined by

$$
\left\{\begin{array}{l}
\vartheta_{00}(0)^{2} x_{0}^{2}=\vartheta_{01}(0)^{2} x_{1}^{2}+\vartheta_{10}(0)^{2} x_{2}^{2} \\
\vartheta_{00}(0)^{2} x_{3}^{2}=\vartheta_{10}(0)^{2} x_{1}^{2}-\vartheta_{01}(0)^{2} x_{2}^{2}
\end{array} .\right.
$$

This is proved using the addition theorem of theta functions ([8]).

\section{§2. Chow-forms}

In this section, by using the classical elimination theory, we compute the Chow-form of the curve $X_{\tau}$ which is defined by (1). Since the Chow-form $P_{X}(U)$ 
of a projective variety $X$ of dimension $r$ and of degree $m$ in a projective space $\boldsymbol{P}^{n}$ can be expressed as a homogeneous polynomial of degree $m$ in the determinants

$$
\left|\begin{array}{lll}
U_{0 i_{0}} & \cdots & U_{0 i_{r}} \\
& \cdots & \\
U_{r i_{0}} & \cdots & U_{r i_{r}}
\end{array}\right|
$$

with sequences of indeterminates $U_{\alpha}=\left(U_{\alpha 0}, \ldots, U_{\alpha n}\right)(0 \leq \alpha \leq r)$ ([2] or [4] or [7]), we shall express the Chow-form of $X_{\tau}$ as a homogeneous polynomial in the determinants as above of the indeterminates, whose coefficients are theta constants.

2.1. In this item, forgetting (1), a little generally, we consider the Chow-form corresponding to our case.

Let $a_{0}, a_{1}, a_{2}$ be non-zero elements of $C^{\times}=C-\{0\}$. We consider two quadratic forms $f_{1}, f_{2}$ :

$$
\begin{aligned}
& f_{1}:=a_{0}^{2} x_{0}^{2}-a_{1}^{2} x_{1}^{2}-a_{2}^{2} x_{2}^{2}, \\
& f_{2}:=a_{0}^{2} x_{3}^{2}-a_{2}^{2} x_{1}^{2}+a_{1}^{2} x_{2}^{2} .
\end{aligned}
$$

Let $V\left(f_{1}\right), V\left(f_{2}\right)$ be the quadrics in $\boldsymbol{P}^{3}$ defined by $f_{1}=0, f_{2}=0$.

As to the irreducibility of an algebraic subset $V\left(f_{1}\right) \cap V\left(f_{2}\right)$ in $\boldsymbol{P}^{3}$, we have

Proposition. Let $\Sigma$ be the pencil of quadrics defined by $V\left(f_{1}\right)$ and $V\left(f_{2}\right)$. If

$$
a_{0}^{4}=a_{1}^{4}+a_{2}^{4}
$$

then $\Sigma$ is separable. Hence, the intersection $V\left(f_{1}\right) \cap V\left(f_{2}\right)$ is an irreducible curve of degree 4 in $\boldsymbol{P}^{3}$.

The first part is proved along the analogous way as [9], pp. $73 \sim 74$, and the irreducibility of the intersection $V\left(f_{1}\right) \cap V\left(f_{2}\right)$ is a direct conclusion of the separability of $\Sigma([9])$.

Therefore, in that case, the Chow-form of the curve $V\left(f_{1}\right) \cap V\left(f_{2}\right)$ is irreducible.

Now, assuming that $a_{0}, a_{1}$ and $a_{2}$ satisfy (2), we compute the Chow-form of the curve $X=V\left(f_{1}\right) \cap V\left(f_{2}\right)$.

Let

$$
u^{(0)}=\left(u_{0}^{(0)}, u_{1}^{(0)}, u_{2}^{(0)}, u_{3}^{(0)}\right), \quad u^{(1)}=\left(u_{0}^{(1)}, u_{1}^{(1)}, u_{2}^{(1)}, u_{3}^{(1)}\right)
$$

be two sequences of independent variables. 
We consider the following system of equations:

$$
\left\{\begin{array}{l}
f_{1}:=a_{0}^{2} x_{0}^{2}-a_{1}^{2} x_{1}^{2}-a_{2}^{2} x_{2}^{2}=0 \\
f_{2}:=a_{0}^{2} x_{3}^{2}-a_{2}^{2} x_{1}^{2}+a_{1}^{2} x_{2}^{2}=0 \\
f_{3} \equiv u_{0}^{(0)} x_{0}+\cdots+u_{3}^{(0)} x_{3}=0 \\
f_{4} \equiv u_{0}^{(1)} x_{0}+\cdots+u_{3}^{(1)} x_{3}=0 .
\end{array}\right.
$$

Then a G.C.D. of the resutant system $\mathscr{R}$ of the system of equations (3) is a power of the Chow-form $P_{X}$ of $X([10])$.

In our case, by A. Hurwitz ([3]), the resutant system $\mathscr{R}$ consists of one resultant which is a G.C.D. of Trägheitsformen of (3). But, as the following procedure shows, if we use the Plücker coordinates of the independent variables, then we can perform the elimination using only Sylvester's determinant without A. Hurwitz's theorem.

We put

$$
p_{i j}=u_{i}^{(0)} u_{j}^{(1)}-u_{j}^{(0)} u_{i}^{(1)} \quad(0 \leq i<j \leq 3)
$$

Plücker's relation is

$$
p_{03} p_{12}=p_{02} p_{13}-p_{01} p_{23}
$$

Put $s=x_{2}, t=x_{3}$.

From $f_{3}=f_{4}=0$, we get

$$
\left\{\begin{array}{l}
p_{01} x_{0}=s p_{12}+t p_{13} \\
p_{01} x_{1}=-s p_{02}-t p_{03}
\end{array}\right.
$$

We eliminate $x_{0}, x_{1}$ from $f_{1}, f_{2}$.

First we have

$$
\begin{aligned}
& p_{01}^{2} f_{1}=\left(a_{0}^{2} p_{12}^{2}-a_{1}^{2} p_{02}^{2}-a_{2}^{2} p_{01}^{2}\right) s^{2}+2\left(a_{0}^{2} p_{12} p_{13}-a_{1}^{2} p_{02} p_{03}\right) s t+\left(a_{0}^{2} p_{13}^{2}-a_{1}^{2} p_{03}^{2}\right) t^{2} \\
& p_{01}^{2} f_{2}=\left(a_{1}^{2} p_{01}^{2}-a_{2}^{2} p_{02}^{2}\right) s^{2}-2 a_{2}^{2} p_{02} p_{03} s t+\left(a_{0}^{2} p_{01}^{2}-a_{2}^{2} p_{03}^{2}\right) t^{2}
\end{aligned}
$$

We define

$$
\begin{aligned}
F_{1} & :=\left(a_{0}^{2} p_{12}^{2}-a_{1}^{2} p_{02}^{2}-a_{2}^{2} p_{01}^{2}\right) s^{2}+2\left(a_{0}^{2} p_{12} p_{13}-a_{1}^{2} p_{02} p_{03}\right) s t+\left(a_{0}^{2} p_{13}^{2}-a_{1}^{2} p_{03}^{2}\right) t^{2} \\
& \stackrel{\text { put }}{=} c_{0} s^{2}+c_{1} s t+c_{2} t^{2} \\
F_{2} & :=\left(a_{1}^{2} p_{01}^{2}-a_{2}^{2} p_{02}^{2}\right) s^{2}-2 a_{2}^{2} p_{02} p_{03} s t+\left(a_{0}^{2} p_{01}^{2}-a_{2}^{2} p_{03}^{2}\right) t^{2} \\
& \stackrel{\text { put }}{=} d_{0} s^{2}+d_{1} s t+d_{2} t^{2}
\end{aligned}
$$


i.e.,

$$
\left\{\begin{array}{l}
c_{0}=a_{0}^{2} p_{12}^{2}-a_{1}^{2} p_{02}^{2}-a_{2}^{2} p_{01}^{2} \\
c_{1}=2\left(a_{0}^{2} p_{12} p_{13}-a_{1}^{2} p_{02} p_{03}\right) \\
c_{2}=a_{0}^{2} p_{13}^{2}-a_{1}^{2} p_{03}^{2} \\
d_{0}=a_{1}^{2} p_{01}^{2}-a_{2}^{2} p_{02}^{2} \\
d_{1}=-2 a_{2}^{2} p_{02} p_{03} \\
d_{2}=a_{0}^{2} p_{01}^{2}-a_{2}^{2} p_{03}^{2}
\end{array} .\right.
$$

Now, we consider the system of equations:

$$
\left\{\begin{array}{l}
F_{1}=c_{0} s^{2}+c_{1} s t+c_{2} t^{2}=0 \\
F_{2}=d_{0} s^{2}+d_{1} s t+d_{2} t^{2}=0
\end{array} .\right.
$$

Then we have the equivalence: (3) has a non-trivial solution $\Leftrightarrow(5)$ has a nontrivial solution.

Let $R\left(F_{1}, F_{2}\right)$ be the Sylvester's determinant of (5), i.e.,

$$
\begin{aligned}
R\left(F_{1}, F_{2}\right) & =\left(c_{0} d_{2}-c_{2} d_{0}\right)^{2}+\left(c_{0} d_{1}-d_{0} c_{1}\right)\left(c_{2} d_{1}-d_{2} c_{1}\right) \\
& =c_{2}^{2} d_{0}^{2}-c_{1} c_{2} d_{0} d_{1}+c_{0} c_{2} d_{1}^{2}+c_{1}^{2} d_{0} d_{2}-2 c_{0} c_{2} d_{0} d_{2}-c_{0} c_{1} d_{1} d_{2}+c_{0}^{2} d_{2}^{2} .
\end{aligned}
$$

Now, using (b), we compute each term in the right side of (c).

$$
\begin{aligned}
c_{2}^{2} d_{0}^{2}= & a_{1}^{8} p_{01}^{4} p_{03}^{4}-2 a_{1}^{6} a_{2}^{2} p_{01}^{2} p_{02}^{2} p_{03}^{4}+a_{1}^{4} a_{2}^{4} p_{02}^{4} p_{03}^{4}-2 a_{0}^{2} a_{1}^{6} p_{01}^{4} p_{03}^{2} p_{13}^{2} \\
+ & 4 a_{0}^{2} a_{1}^{4} a_{2}^{2} p_{01}^{2} p_{02}^{2} p_{03}^{2} p_{13}^{2}-2 a_{0}^{2} a_{1}^{2} a_{2}^{4} p_{02}^{4} p_{03}^{2} p_{13}^{2}+a_{0}^{4} a_{1}^{4} p_{01}^{4} p_{13}^{4} \\
- & 2 a_{0}^{4} a_{1}^{2} a_{2}^{2} p_{01}^{2} p_{02}^{2} p_{13}^{4}+a_{0}^{4} a_{2}^{4} p_{02}^{4} p_{13}^{4} . \\
c_{1} c_{2} d_{0} d_{1}= & -4 a_{1}^{6} a_{2}^{2} p_{01}^{2} p_{02}^{2} p_{03}^{4}+4 a_{1}^{4} a_{2}^{4} p_{02}^{4} p_{03}^{4} \\
& +4 a_{0}^{2} a_{1}^{4} a_{2}^{2} p_{01}^{2} p_{02} p_{03}^{3} p_{12} p_{13}-4 a_{0}^{2} a_{1}^{2} a_{2}^{4} p_{02}^{3} p_{03}^{3} p_{12} p_{13} \\
& +4 a_{0}^{2} a_{1}^{4} a_{2}^{2} p_{01}^{2} p_{02}^{2} p_{03}^{2} p_{13}^{2}-4 a_{0}^{2} a_{1}^{2} a_{2}^{4} p_{02}^{4} p_{03}^{2} p_{13}^{2} \\
& -4 a_{0}^{4} a_{1}^{2} a_{2}^{2} p_{01}^{2} p_{02} p_{03} p_{12} p_{13}^{3}+4 a_{0}^{4} a_{2}^{4} p_{02}^{3} p_{03} p_{12} p_{13}^{3} . \\
c_{0} c_{2} d_{1}^{2}= & 4 a_{1}^{2} a_{2}^{6} p_{01}^{2} p_{02}^{2} p_{03}^{4}+4 a_{1}^{4} a_{2}^{4} p_{02}^{4} p_{03}^{4}-4 a_{0}^{2} a_{1}^{2} a_{2}^{4} p_{02}^{2} p_{03}^{4} p_{12}^{2} \\
& -4 a_{0}^{2} a_{2}^{6} p_{01}^{2} p_{02}^{2} p_{03}^{2} p_{13}^{2}-4 a_{0}^{2} a_{1}^{2} a_{2}^{4} p_{02}^{4} p_{03}^{2} p_{13}^{2} \\
& +4 a_{0}^{4} a_{2}^{4} p_{02}^{2} p_{03}^{2} p_{12}^{2} p_{13}^{2} .
\end{aligned}
$$




$$
\begin{aligned}
c_{1}^{2} d_{0} d_{2}= & 4 a_{0}^{2} a_{1}^{6} p_{01}^{4} p_{02}^{2} p_{03}^{2}-4 a_{0}^{2} a_{1}^{4} a_{2}^{2} p_{01}^{2} p_{02}^{4} p_{03}^{2} \\
& -4 a_{1}^{6} a_{2}^{2} p_{01}^{2} p_{02}^{2} p_{03}^{4}+4 a_{1}^{4} a_{2}^{4} p_{02}^{4} p_{03}^{4}-8 a_{0}^{4} a_{1}^{4} p_{01}^{4} p_{02} p_{03} p_{12} p_{13} \\
& +8 a_{0}^{4} a_{1}^{2} a_{2}^{2} p_{01}^{2} p_{02}^{3} p_{03} p_{12} p_{13}+8 a_{0}^{2} a_{1}^{4} a_{2}^{2} p_{01}^{2} p_{02} p_{03}^{3} p_{12} p_{13} \\
& -8 a_{0}^{2} a_{1}^{2} a_{2}^{4} p_{02}^{3} p_{03}^{3} p_{12} p_{13}+4 a_{0}^{6} a_{1}^{2} p_{01}^{4} p_{12}^{2} p_{13}^{2}-4 a_{0}^{6} a_{2}^{2} p_{01}^{2} p_{02}^{2} p_{12}^{2} p_{13}^{2} \\
- & 4 a_{0}^{4} a_{1}^{2} a_{2}^{2} p_{01}^{2} p_{03}^{2} p_{12}^{2} p_{13}^{2}+4 a_{0}^{4} a_{2}^{4} p_{02}^{2} p_{03}^{2} p_{12}^{2} p_{13}^{2} . \\
c_{0} c_{2} d_{0} d_{2}= & a_{0}^{2} a_{1}^{4} a_{2}^{2} p_{01}^{6} p_{03}^{2}+a_{0}^{2} a_{1}^{6} p_{01}^{4} p_{02}^{2} p_{03}^{2}-a_{0}^{2} a_{1}^{2} a_{2}^{4} p_{01}^{4} p_{02}^{2} p_{03}^{2} \\
& -a_{0}^{2} a_{1}^{4} a_{2}^{2} p_{01}^{2} p_{02}^{4} p_{03}^{2}-a_{1}^{4} a_{2}^{4} p_{01}^{4} p_{03}^{4}-a_{1}^{6} a_{2}^{2} p_{01}^{2} p_{02}^{2} p_{03}^{4} \\
& +a_{1}^{2} a_{2}^{6} p_{01}^{2} p_{02}^{2} p_{03}^{4}+a_{1}^{4} a_{2}^{4} p_{02}^{4} p_{03}^{4}-a_{0}^{4} a_{1}^{4} p_{01}^{4} p_{03}^{2} p_{12}^{2} \\
& +a_{0}^{4} a_{1}^{2} a_{2}^{2} p_{01}^{2} p_{02}^{2} p_{03}^{2} p_{12}^{2}+a_{0}^{2} a_{1}^{4} a_{2}^{2} p_{01}^{2} p_{03}^{4} p_{12}^{2}-a_{0}^{2} a_{1}^{2} a_{2}^{4} p_{02}^{2} p_{03}^{4} p_{12}^{2} \\
& -a_{0}^{4} a_{1}^{2} a_{2}^{2} p_{01}^{6} p_{13}^{2}-a_{0}^{4} a_{1}^{4} p_{01}^{4} p_{02}^{2} p_{13}^{2}+a_{0}^{4} a_{2}^{4} p_{01}^{4} p_{02}^{2} p_{13}^{2} \\
& +a_{0}^{4} a_{1}^{2} a_{2}^{2} p_{01}^{2} p_{02}^{4} p_{13}^{2}+a_{0}^{2} a_{1}^{2} a_{2}^{4} p_{01}^{4} p_{03}^{2} p_{13}^{2}+a_{0}^{2} a_{1}^{4} a_{2}^{2} p_{01}^{2} p_{02}^{2} p_{03}^{2} p_{13}^{2} \\
& -a_{0}^{2} a_{2}^{6} p_{01}^{2} p_{02}^{2} p_{03}^{2} p_{13}^{2}-a_{0}^{2} a_{1}^{2} a_{2}^{4} p_{02}^{4} p_{03}^{2} p_{13}^{2}+a_{0}^{6} a_{1}^{2} p_{01}^{4} p_{12}^{2} p_{13}^{2} \\
& -a_{0}^{6} a_{2}^{2} p_{01}^{2} p_{02}^{2} p_{12}^{2} p_{13}^{2}-a_{0}^{4} a_{1}^{2} a_{2}^{2} p_{01}^{2} p_{03}^{2} p_{12}^{2} p_{13}^{2} \\
& +a_{0}^{4} a_{2}^{4} p_{02}^{2} p_{03}^{2} p_{12}^{2} p_{13}^{2} . \\
&
\end{aligned}
$$

$c_{0} c_{1} d_{1} d_{2}=-4 a_{0}^{2} a_{1}^{2} a_{2}^{4} p_{01}^{4} p_{02}^{2} p_{03}^{2}-4 a_{0}^{2} a_{1}^{4} a_{2}^{2} p_{01}^{2} p_{02}^{4} p_{03}^{2}$

$$
\begin{aligned}
& +4 a_{1}^{2} a_{2}^{6} p_{01}^{2} p_{02}^{2} p_{03}^{4}+4 a_{1}^{4} a_{2}^{4} p_{02}^{4} p_{03}^{4}+4 a_{0}^{4} a_{1}^{2} a_{2}^{2} p_{01}^{2} p_{02}^{2} p_{03}^{2} p_{12}^{2} \\
& -4 a_{0}^{2} a_{1}^{2} a_{2}^{4} p_{02}^{2} p_{03}^{4} p_{12}^{2}+4 a_{0}^{4} a_{2}^{4} p_{01}^{4} p_{02} p_{03} p_{12} p_{13} \\
& +4 a_{0}^{4} a_{1}^{2} a_{2}^{2} p_{01}^{2} p_{02}^{3} p_{03} p_{12} p_{13}-4 a_{0}^{2} a_{2}^{6} p_{01}^{2} p_{02} p_{03}^{3} p_{12} p_{13} \\
& -4 a_{0}^{2} a_{1}^{2} a_{2}^{4} p_{02}^{3} p_{03}^{3} p_{12} p_{13}-4 a_{0}^{6} a_{2}^{2} p_{01}^{2} p_{02} p_{03} p_{12}^{3} p_{13} \\
& +4 a_{0}^{4} a_{2}^{4} p_{02} p_{03}^{3} p_{12}^{3} p_{13} .
\end{aligned}
$$$$
c_{0}^{2} d_{2}^{2}=a_{0}^{4} a_{2}^{4} p_{01}^{8}+2 a_{0}^{4} a_{1}^{2} a_{2}^{2} p_{01}^{6} p_{02}^{2}+a_{0}^{4} a_{1}^{4} p_{01}^{4} p_{02}^{4}-2 a_{0}^{2} a_{2}^{6} p_{01}^{6} p_{03}^{2}
$$$$
-4 a_{0}^{2} a_{1}^{2} a_{2}^{4} p_{01}^{4} p_{02}^{2} p_{03}^{2}-2 a_{0}^{2} a_{1}^{4} a_{2}^{2} p_{01}^{2} p_{02}^{4} p_{03}^{2}+a_{2}^{8} p_{01}^{4} p_{03}^{4}
$$$$
+2 a_{1}^{2} a_{2}^{6} p_{01}^{2} p_{02}^{2} p_{03}^{4}+a_{1}^{4} a_{2}^{4} p_{02}^{4} p_{03}^{4}-2 a_{0}^{6} a_{2}^{2} p_{01}^{6} p_{12}^{2}-2 a_{0}^{6} a_{1}^{2} p_{01}^{4} p_{02}^{2} p_{12}^{2}
$$$$
+4 a_{0}^{4} a_{2}^{4} p_{01}^{4} p_{03}^{2} p_{12}^{2}+4 a_{0}^{4} a_{1}^{2} a_{2}^{2} p_{01}^{2} p_{02}^{2} p_{03}^{2} p_{12}^{2}-2 a_{0}^{2} a_{2}^{6} p_{01}^{2} p_{03}^{4} p_{12}^{2}
$$ 


$$
\begin{aligned}
& -2 a_{0}^{2} a_{1}^{2} a_{2}^{4} p_{02}^{2} p_{03}^{4} p_{12}^{2}+a_{0}^{8} p_{01}^{4} p_{12}^{4}-2 a_{0}^{6} a_{2}^{2} p_{01}^{2} p_{03}^{2} p_{12}^{4} \\
& +a_{0}^{4} a_{2}^{4} p_{03}^{4} p_{12}^{4}
\end{aligned}
$$

By substituting $(\mathrm{c} 1), \ldots,(\mathrm{c} 7)$ into the right side of $(\mathrm{c})$, we have

$$
\begin{aligned}
& R\left(F_{1}, F_{2}\right)=c_{2}^{2} d_{0}^{2}-c_{1} c_{2} d_{0} d_{1}+c_{0} c_{2} d_{1}^{2}+c_{1}^{2} d_{0} d_{2}-2 c_{0} c_{2} d_{0} d_{2}-c_{0} c_{1} d_{1} d_{2}+c_{0}^{2} d_{2}^{2} \\
& =\left\{a_{1}^{8} p_{01}^{4} p_{03}^{4}-2 a_{1}^{6} a_{2}^{2} p_{01}^{2} p_{02}^{2} p_{03}^{4}+a_{1}^{4} a_{2}^{4} p_{02}^{4} p_{03}^{4}-2 a_{0}^{2} a_{1}^{6} p_{01}^{4} p_{03}^{2} p_{13}^{2}\right. \\
& +4 a_{0}^{2} a_{1}^{4} a_{2}^{2} p_{01}^{2} p_{02}^{2} p_{03}^{2} p_{13}^{2}-2 a_{0}^{2} a_{1}^{2} a_{2}^{4} p_{02}^{4} p_{03}^{2} p_{13}^{2}+a_{0}^{4} a_{1}^{4} p_{01}^{4} p_{13}^{4} \\
& \left.-2 a_{0}^{4} a_{1}^{2} a_{2}^{2} p_{01}^{2} p_{02}^{2} p_{13}^{4}+a_{0}^{4} a_{2}^{4} p_{02}^{4} p_{13}^{4}\right\} \\
& -\left\{-4 a_{1}^{6} a_{2}^{2} p_{01}^{2} p_{02}^{2} p_{03}^{4}+4 a_{1}^{4} a_{2}^{4} p_{02}^{4} p_{03}^{4}\right. \\
& -4 a_{0}^{2} a_{1}^{4} a_{2}^{2} p_{01}^{3} p_{02} p_{03}^{2} p_{13} p_{23}+4 a_{0}^{2} a_{1}^{2} a_{2}^{4} p_{01} p_{02}^{3} p_{03}^{2} p_{13} p_{23} \\
& +8 a_{0}^{2} a_{1}^{4} a_{2}^{2} p_{01}^{2} p_{02}^{2} p_{03}^{2} p_{13}^{2}-8 a_{0}^{2} a_{1}^{2} a_{2}^{4} p_{02}^{4} p_{03}^{2} p_{13}^{2} \\
& \left.-4 a_{0}^{4} a_{1}^{2} a_{2}^{2} p_{01}^{2} p_{02}\left(p_{02} p_{13}-p_{01} p_{23}\right) p_{13}^{3}+4 a_{0}^{4} a_{2}^{4} p_{02}^{3}\left(p_{02} p_{13}-p_{01} p_{23}\right) p_{13}^{3}\right\} \\
& +\left\{4 a_{1}^{2} a_{2}^{6} p_{01}^{2} p_{02}^{2} p_{03}^{4}+4 a_{1}^{4} a_{2}^{4} p_{02}^{4} p_{03}^{4}\right. \\
& -4 a_{0}^{2} a_{1}^{2} a_{2}^{4} p_{02}^{2} p_{03}^{2}\left(-2 p_{01} p_{02} p_{13} p_{23}+p_{01}^{2} p_{23}^{2}\right) \\
& -4 a_{0}^{2} a_{2}^{6} p_{01}^{2} p_{02}^{2} p_{03}^{2} p_{13}^{2}-8 a_{0}^{2} a_{1}^{2} a_{2}^{4} p_{02}^{4} p_{03}^{2} p_{13}^{2} \\
& \left.+4 a_{0}^{4} a_{2}^{4} p_{02}^{2}\left(p_{02}^{2} p_{13}^{2}-2 p_{01} p_{02} p_{13} p_{23}+p_{01}^{2} p_{23}^{2}\right) p_{13}^{2}\right\} \\
& +\left\{4 a_{0}^{2} a_{1}^{6} p_{01}^{4} p_{02}^{2} p_{03}^{2}-4 a_{0}^{2} a_{1}^{4} a_{2}^{2} p_{01}^{2} p_{02}^{4} p_{03}^{2}\right. \\
& -4 a_{1}^{6} a_{2}^{2} p_{01}^{2} p_{02}^{2} p_{03}^{4}+4 a_{1}^{4} a_{2}^{4} p_{02}^{4} p_{03}^{4}-8 a_{0}^{4} a_{1}^{4} p_{01}^{4} p_{02}\left(p_{02} p_{13}-p_{01} p_{23}\right) p_{13} \\
& +8 a_{0}^{4} a_{1}^{2} a_{2}^{2} p_{01}^{2} p_{02}^{3}\left(p_{02} p_{13}-p_{01} p_{23}\right) p_{13} \\
& +8 a_{0}^{2} a_{1}^{4} a_{2}^{2} p_{01}^{2} p_{02} p_{03}^{2}\left(p_{02} p_{13}-p_{01} p_{23}\right) p_{13} \\
& -8 a_{0}^{2} a_{1}^{2} a_{2}^{4} p_{02}^{3} p_{03}^{2}\left(p_{02} p_{13}-p_{01} p_{23}\right) p_{13}+4 a_{0}^{6} a_{1}^{2} p_{01}^{4} p_{12}^{2} p_{13}^{2} \\
& -4 a_{0}^{6} a_{2}^{2} p_{01}^{2} p_{02}^{2} p_{12}^{2} p_{13}^{2}-4 a_{0}^{4} a_{1}^{2} a_{2}^{2} p_{01}^{2}\left(p_{02}^{2} p_{13}^{2}-2 p_{01} p_{02} p_{13} p_{23}+p_{01}^{2} p_{23}^{2}\right) p_{13}^{2} \\
& \left.+4 a_{0}^{4} a_{2}^{4} p_{02}^{2}\left(p_{02}^{2} p_{13}^{2}-2 p_{01} p_{02} p_{13} p_{23}+p_{01}^{2} p_{23}^{2}\right) p_{13}^{2}\right\} \\
& -2\left\{a_{0}^{2} a_{1}^{4} a_{2}^{2} p_{01}^{6} p_{03}^{2}+a_{0}^{2} a_{1}^{6} p_{01}^{4} p_{02}^{2} p_{03}^{2}-a_{0}^{2} a_{1}^{2} a_{2}^{4} p_{01}^{4} p_{02}^{2} p_{03}^{2}\right. \\
& -a_{0}^{2} a_{1}^{4} a_{2}^{2} p_{01}^{2} p_{02}^{4} p_{03}^{2}-a_{1}^{4} a_{2}^{4} p_{01}^{4} p_{03}^{4}-a_{1}^{6} a_{2}^{2} p_{01}^{2} p_{02}^{2} p_{03}^{4}
\end{aligned}
$$




$$
\begin{aligned}
& +a_{1}^{2} a_{2}^{6} p_{01}^{2} p_{02}^{2} p_{03}^{4}+a_{1}^{4} a_{2}^{4} p_{02}^{4} p_{03}^{4} \\
& -a_{0}^{4} a_{1}^{4} p_{01}^{4}\left(p_{02}^{2} p_{13}^{2}-2 p_{01} p_{02} p_{13} p_{23}+p_{01}^{2} p_{23}^{2}\right) \\
& +a_{0}^{4} a_{1}^{2} a_{2}^{2} p_{01}^{2} p_{02}^{2}\left(p_{02}^{2} p_{13}^{2}-2 p_{01} p_{02} p_{13} p_{23}+p_{01}^{2} p_{23}^{2}\right) \\
& +a_{0}^{2} a_{1}^{4} a_{2}^{2} p_{01}^{2} p_{03}^{2}\left(p_{02}^{2} p_{13}^{2}-2 p_{01} p_{02} p_{13} p_{23}+p_{01}^{2} p_{23}^{2}\right) \\
& -a_{0}^{2} a_{1}^{2} a_{2}^{4} p_{02}^{2} p_{03}^{2}\left(p_{02}^{2} p_{13}^{2}-2 p_{01} p_{02} p_{13} p_{23}+p_{01}^{2} p_{23}^{2}\right) \\
& -a_{0}^{4} a_{1}^{2} a_{2}^{2} p_{01}^{6} p_{13}^{2}-a_{0}^{4} a_{1}^{4} p_{01}^{4} p_{02}^{2} p_{13}^{2}+a_{0}^{4} a_{2}^{4} p_{01}^{4} p_{02}^{2} p_{13}^{2} \\
& +a_{0}^{4} a_{1}^{2} a_{2}^{2} p_{01}^{2} p_{02}^{4} p_{13}^{2}+a_{0}^{2} a_{1}^{2} a_{2}^{4} p_{01}^{4} p_{03}^{2} p_{13}^{2}+a_{0}^{2} a_{1}^{4} a_{2}^{2} p_{01}^{2} p_{02}^{2} p_{03}^{2} p_{13}^{2} \\
& -a_{0}^{2} a_{2}^{6} p_{01}^{2} p_{02}^{2} p_{03}^{2} p_{13}^{2}-a_{0}^{2} a_{1}^{2} a_{2}^{4} p_{02}^{4} p_{03}^{2} p_{13}^{2}+a_{0}^{6} a_{1}^{2} p_{01}^{4} p_{12}^{2} p_{13}^{2} \\
& -a_{0}^{6} a_{2}^{2} p_{01}^{2} p_{02}^{2} p_{12}^{2} p_{13}^{2}-a_{0}^{4} a_{1}^{2} a_{2}^{2} p_{01}^{2}\left(p_{02}^{2} p_{13}^{2}-2 p_{01} p_{02} p_{13} p_{23}+p_{01}^{2} p_{23}^{2}\right) p_{13}^{2} \\
& \left.+a_{0}^{4} a_{2}^{4} p_{02}^{2}\left(p_{02}^{2} p_{13}^{2}-2 p_{01} p_{02} p_{13} p_{23}+p_{01}^{2} p_{23}^{2}\right) p_{13}^{2}\right\} \\
& -\left\{-4 a_{0}^{2} a_{1}^{2} a_{2}^{4} p_{01}^{4} p_{02}^{2} p_{03}^{2}-4 a_{0}^{2} a_{1}^{4} a_{2}^{2} p_{01}^{2} p_{02}^{4} p_{03}^{2}\right. \\
& +4 a_{1}^{2} a_{2}^{6} p_{01}^{2} p_{02}^{2} p_{03}^{4}+4 a_{1}^{4} a_{2}^{4} p_{02}^{4} p_{03}^{4} \\
& +4 a_{0}^{4} a_{1}^{2} a_{2}^{2} p_{01}^{2} p_{02}^{2}\left(p_{02}^{2} p_{13}^{2}-2 p_{01} p_{02} p_{13} p_{23}+p_{01}^{2} p_{23}^{2}\right) \\
& -4 a_{0}^{2} a_{1}^{2} a_{2}^{4} p_{02}^{2} p_{03}^{2}\left(p_{02}^{2} p_{13}^{2}-2 p_{01} p_{02} p_{13} p_{23}+p_{01}^{2} p_{23}^{2}\right) \\
& +4 a_{0}^{4} a_{2}^{4} p_{01}^{4} p_{02}\left(p_{02} p_{13}-p_{01} p_{23}\right) p_{13} \\
& +4 a_{0}^{4} a_{1}^{2} a_{2}^{2} p_{01}^{2} p_{02}^{3}\left(p_{02} p_{13}-p_{01} p_{23}\right) p_{13} \\
& -4 a_{0}^{2} a_{2}^{6} p_{01}^{2} p_{02} p_{03}^{2}\left(p_{02} p_{13}-p_{01} p_{23}\right) p_{13} \\
& -4 a_{0}^{2} a_{1}^{2} a_{2}^{4} p_{02}^{3} p_{03}^{2}\left(p_{02} p_{13}-p_{01} p_{23}\right) p_{13} \\
& -4 a_{0}^{6} a_{2}^{2} p_{01}^{2} p_{02}\left(p_{02} p_{13}-p_{01} p_{23}\right) p_{12}^{2} p_{13} \\
& \left.+4 a_{0}^{4} a_{2}^{4} p_{02}\left(p_{02}^{3} p_{13}^{3}-3 p_{01} p_{02}^{2} p_{13}^{2} p_{23}+3 p_{01}^{2} p_{02} p_{13} p_{23}^{2}-p_{01}^{3} p_{23}^{3}\right) p_{13}\right\} \\
& +\left\{a_{0}^{4} a_{2}^{4} p_{01}^{8}+2 a_{0}^{4} a_{1}^{2} a_{2}^{2} p_{01}^{6} p_{02}^{2}+a_{0}^{4} a_{1}^{4} p_{01}^{4} p_{02}^{4}-2 a_{0}^{2} a_{2}^{6} p_{01}^{6} p_{03}^{2}\right. \\
& -4 a_{0}^{2} a_{1}^{2} a_{2}^{4} p_{01}^{4} p_{02}^{2} p_{03}^{2}-2 a_{0}^{2} a_{1}^{4} a_{2}^{2} p_{01}^{2} p_{02}^{4} p_{03}^{2}+a_{2}^{8} p_{01}^{4} p_{03}^{4} \\
& +2 a_{1}^{2} a_{2}^{6} p_{01}^{2} p_{02}^{2} p_{03}^{4}+a_{1}^{4} a_{2}^{4} p_{02}^{4} p_{03}^{4}-2 a_{0}^{6} a_{2}^{2} p_{01}^{6} p_{12}^{2}-2 a_{0}^{6} a_{1}^{2} p_{01}^{4} p_{02}^{2} p_{12}^{2} \\
& +4 a_{0}^{4} a_{2}^{4} p_{01}^{4}\left(p_{02}^{2} p_{13}^{2}-2 p_{01} p_{02} p_{13} p_{23}+p_{01}^{2} p_{23}^{2}\right)
\end{aligned}
$$




$$
\begin{aligned}
& +4 a_{0}^{4} a_{1}^{2} a_{2}^{2} p_{01}^{2} p_{02}^{2}\left(p_{02}^{2} p_{13}^{2}-2 p_{01} p_{02} p_{13} p_{23}+p_{01}^{2} p_{23}^{2}\right) \\
& -2 a_{0}^{2} a_{2}^{6} p_{01}^{2} p_{03}^{2}\left(p_{02}^{2} p_{13}^{2}-2 p_{01} p_{02} p_{13} p_{23}+p_{01}^{2} p_{23}^{2}\right) \\
& -2 a_{0}^{2} a_{1}^{2} a_{2}^{4} p_{02}^{2} p_{03}^{2}\left(p_{02}^{2} p_{13}^{2}-2 p_{01} p_{02} p_{13} p_{23}+p_{01}^{2} p_{23}^{2}\right)+a_{0}^{8} p_{01}^{4} p_{12}^{4} \\
& -2 a_{0}^{6} a_{2}^{2} p_{01}^{2}\left(p_{02}^{2} p_{13}^{2}-2 p_{01} p_{02} p_{13} p_{23}+p_{01}^{2} p_{23}^{2}\right) p_{12}^{2} \\
& +a_{0}^{4} a_{2}^{4}\left(p_{02}^{4} p_{13}^{4}-4 p_{01} p_{02}^{3} p_{13}^{3} p_{23}+6 p_{01}^{2} p_{02}^{2} p_{13}^{2} p_{23}^{2}\right. \\
& \left.\left.-4 p_{01}^{3} p_{02} p_{13} p_{23}^{3}+p_{01}^{4} p_{23}^{4}\right)\right\} .
\end{aligned}
$$

If we use (2) and (a), the right side of (d) is simplified, and we get

$$
\begin{aligned}
R\left(F_{1}, F_{2}\right)= & a_{0}^{2} p_{01}^{4}\left[a_{0}^{4} p_{03}^{4}-2 a_{0}^{2} a_{1}^{2} p_{03}^{2} p_{13}^{2}+a_{1}^{4} p_{13}^{4}+2 a_{0}^{2} a_{1}^{2} p_{02}^{2} p_{03}^{2}\right. \\
& -4 a_{1}^{4} p_{02}^{2} p_{13}^{2}+4 a_{1}^{4} p_{01} p_{02} p_{13} p_{23}+2 a_{0}^{2} a_{1}^{2} p_{12}^{2} p_{13}^{2}-2 a_{1}^{2} a_{2}^{2} p_{23}^{2} p_{13}^{2} \\
& -2 a_{0}^{2} a_{2}^{2} p_{01}^{2} p_{03}^{2}+2 a_{1}^{4} p_{01}^{2} p_{23}^{2}-2 a_{0}^{2} a_{2}^{2} p_{03}^{2} p_{23}^{2}+2 a_{1}^{2} a_{2}^{2} p_{01}^{2} p_{13}^{2} \\
& -2 a_{2}^{4} p_{02}^{2} p_{13}^{2}-2 a_{1}^{2} a_{2}^{2} p_{02}^{2} p_{23}^{2}+a_{2}^{4} p_{01}^{4}+2 a_{1}^{2} a_{2}^{2} p_{01}^{2} p_{02}^{2}+a_{1}^{4} p_{02}^{4} \\
& -2 a_{0}^{2} a_{2}^{2} p_{01}^{2} p_{12}^{2}-2 a_{0}^{2} a_{1}^{2} p_{02}^{2} p_{12}^{2}-4 a_{2}^{4} p_{01} p_{02} p_{13} p_{23}+4 a_{2}^{4} p_{01}^{2} p_{23}^{2} \\
& \left.+a_{0}^{4} p_{12}^{4}-2 a_{0}^{2} a_{2}^{2} p_{12}^{2} p_{23}^{2}+a_{2}^{4} p_{23}^{4}\right] .
\end{aligned}
$$

This is a resultant of (5). The Chow-form $P_{X}$ appears in $R\left(F_{1}, F_{2}\right)$ as a divisor and it is an irreducible homogeneous polynomial of degree 4 in the Plücker coordinates. Now, we take a set of indeterminates $U_{j}^{(i)}$ and denote by capitals $P_{i j}$ the expressions which result if in the Plücker coordinates $p_{i j}$ the $u_{j}^{(i)}$ are replaced by $U_{j}^{(i)}$. Thus we get

THEOREM 1. Let $P_{X}(U)$ be the Chow-form of the curve $X$. Then $P_{X}(U)$ is given by

$$
\begin{aligned}
P_{X}(U)= & a_{0}^{4} P_{03}^{4}-2 a_{0}^{2} a_{1}^{2} P_{03}^{2} P_{13}^{2}+a_{1}^{4} P_{13}^{4}+2 a_{0}^{2} a_{1}^{2} P_{02}^{2} P_{03}^{2}-4 a_{1}^{4} P_{02}^{2} P_{13}^{2} \\
& +4 a_{1}^{4} P_{01} P_{02} P_{13} P_{23}+2 a_{0}^{2} a_{1}^{2} P_{12}^{2} P_{13}^{2}-2 a_{1}^{2} a_{2}^{2} P_{23}^{2} P_{13}^{2}-2 a_{0}^{2} a_{2}^{2} P_{01}^{2} P_{03}^{2} \\
& +2 a_{1}^{4} P_{01}^{2} P_{23}^{2}-2 a_{0}^{2} a_{2}^{2} P_{03}^{2} P_{23}^{2}+2 a_{1}^{2} a_{2}^{2} P_{01}^{2} P_{13}^{2}-2 a_{2}^{4} P_{02}^{2} P_{13}^{2} \\
& -2 a_{1}^{2} a_{2}^{2} P_{02}^{2} P_{23}^{2}+a_{2}^{4} P_{01}^{4}+2 a_{1}^{2} a_{2}^{2} P_{01}^{2} P_{02}^{2}+a_{1}^{4} P_{02}^{4}-2 a_{0}^{2} a_{2}^{2} P_{01}^{2} P_{12}^{2} \\
& -2 a_{0}^{2} a_{1}^{2} P_{02}^{2} P_{12}^{2}-4 a_{2}^{4} P_{01} P_{02} P_{13} P_{23}+4 a_{2}^{4} P_{01}^{2} P_{23}^{2}+a_{0}^{4} P_{12}^{4} \\
& -2 a_{0}^{2} a_{2}^{2} P_{12}^{2} P_{23}^{2}+a_{2}^{4} P_{23}^{4} .
\end{aligned}
$$


2.2. We go back to the situations as in $\S 1$. We fix an arbitrary point $\tau$ of $H$, and so we write simply $X$ instead of $X_{\tau}$.

In the equations $(1)$ in $\S 1$, we put

$$
a_{0}=\vartheta_{00}(0), \quad a_{1}=\vartheta_{01}(0), \quad a_{2}=\vartheta_{10}(0) .
$$

Then

$$
X=V\left(f_{1}\right) \cap V\left(f_{2}\right)
$$

under the notations in 2.1. Moreover, Jacobi's identity is

$$
a_{0}^{4}=a_{1}^{4}+a_{2}^{4} \text {. }
$$

Therefore, from Th. 1, we get

THEOREM 1'. Let $P_{X}(U)$ be the Chow-form of the projective elliptic normal curve $X=\phi\left(E_{\tau}\right)$. Then $P_{X}(U)$ is given by

$$
\begin{aligned}
P_{X}(U)= & \vartheta_{00}(0)^{4} P_{03}^{4}-2 \vartheta_{00}(0)^{2} \vartheta_{01}(0)^{2} P_{03}^{2} P_{13}^{2}+\vartheta_{01}(0)^{4} P_{13}^{4} \\
& +2 \vartheta_{00}(0)^{2} \vartheta_{01}(0)^{2} P_{02}^{2} P_{03}^{2}-4 \vartheta_{01}(0)^{4} P_{02}^{2} P_{13}^{2}+4 \vartheta_{01}(0)^{4} P_{01} P_{02} P_{13} P_{23} \\
& +2 \vartheta_{00}(0)^{2} \vartheta_{01}(0)^{2} P_{12}^{2} P_{13}^{2}-2 \vartheta_{01}(0)^{2} \vartheta_{10}(0)^{2} P_{23}^{2} P_{13}^{2} \\
& -2 \vartheta_{00}(0)^{2} \vartheta_{10}(0)^{2} P_{01}^{2} P_{03}^{2}+2 \vartheta_{01}(0)^{4} P_{01}^{2} P_{23}^{2} \\
& -2 \vartheta_{00}(0)^{2} \vartheta_{10}(0)^{2} P_{03}^{2} P_{23}^{2}+2 \vartheta_{01}(0)^{2} \vartheta_{10}(0)^{2} P_{01}^{2} P_{13}^{2}-2 \vartheta_{10}(0)^{4} P_{02}^{2} P_{13}^{2} \\
& -2 \vartheta_{01}(0)^{2} \vartheta_{10}(0)^{2} P_{02}^{2} P_{23}^{2}+\vartheta_{10}(0)^{4} P_{01}^{4}+2 \vartheta_{01}(0)^{2} \vartheta_{10}(0)^{2} P_{01}^{2} P_{02}^{2} \\
& +\vartheta_{01}(0)^{4} P_{02}^{4}-2 \vartheta_{00}(0)^{2} \vartheta_{10}(0)^{2} P_{01}^{2} P_{12}^{2}-2 \vartheta_{00}(0)^{2} \vartheta_{01}(0)^{2} P_{02}^{2} P_{12}^{2} \\
& -4 \vartheta_{10}(0)^{4} P_{01} P_{02} P_{13} P_{23}+4 \vartheta_{10}(0)^{4} P_{01}^{2} P_{23}^{2}+\vartheta_{00}(0)^{4} P_{12}^{4} \\
& -2 \vartheta_{00}(0)^{2} \vartheta_{10}(0)^{2} P_{12}^{2} P_{23}^{2}+\vartheta_{10}(0)^{4} P_{23}^{4} .
\end{aligned}
$$

Let $G$ be the Grassmann variety of lines in $\boldsymbol{P}^{3}$. By $\Gamma_{4}$, we mean the homogeneous part of degree 4 of the homogeneous coordinate ring $\Gamma$ of $G$. The vector space $\Gamma_{4}$ has a base consisting of the standard monomials of degree 4 , and it is of dimension 105 ([2], p. 387).

The above expression of $P_{X}(U)$ is unique, in the sense that the monomials appearing in the expression are standard.

2.3. We denote by $c(X)$ the Chow point of $X\left(=\phi\left(E_{\tau}\right)\right)$. By the beginning of this $\S, c(X)$ is a point of the projective space $\boldsymbol{P}\left(\Gamma_{4}\right)$. Let $M$ be the linear subspace 
of $\boldsymbol{P}\left(\Gamma_{4}\right)$ spanned by the monomials appearing in the expression of $P_{X}(U)$, i.e.,

$$
\begin{aligned}
M:=\{ & \left\{P_{03}^{4}, P_{03}^{2} P_{13}^{2}, P_{13}^{4}, P_{02}^{2} P_{03}^{2}, P_{02}^{2} P_{13}^{2}, P_{01} P_{02} P_{13} P_{23}, P_{12}^{2} P_{13}^{2}, P_{23}^{2} P_{13}^{2}, P_{01}^{2} P_{03}^{2},\right. \\
& P_{01}^{2} P_{23}^{2}, P_{03}^{2} P_{23}^{2}, P_{01}^{2} P_{13}^{2}, P_{02}^{2} P_{13}^{2}, P_{02}^{2} P_{23}^{2}, P_{01}^{4}, P_{01}^{2} P_{02}^{2}, P_{02}^{4}, P_{01}^{2} P_{12}^{2}, P_{02}^{2} P_{12}^{2}, \\
& \left.\left.P_{01} P_{02} P_{13} P_{23}, P_{01}^{2} P_{23}^{2}, P_{12}^{4}, P_{12}^{2} P_{23}^{2}, P_{23}^{4}\right\}\right\} .
\end{aligned}
$$

Then the theorem shows that:

(1) $M$ contains not only $c(X)$, but also $c\left(\phi\left(E_{\tau}\right)\right)$ for $\forall \tau \in H$,

(2) identifying $M$ with $\boldsymbol{P}^{23}$, a system of homogeneous coordinates of $c(X)$ is given by

$$
\begin{aligned}
c(X)= & \left(\vartheta_{00}(0)^{4},-2 \vartheta_{00}(0)^{2} \vartheta_{01}(0)^{2}, \vartheta_{01}(0)^{4}, 2 \vartheta_{00}(0)^{2} \vartheta_{01}(0)^{2},-4 \vartheta_{01}(0)^{4}, 4 \vartheta_{01}(0)^{4},\right. \\
& 2 \vartheta_{00}(0)^{2} \vartheta_{01}(0)^{2},-2 \vartheta_{01}(0)^{2} \vartheta_{10}(0)^{2},-2 \vartheta_{00}(0)^{2} \vartheta_{10}(0)^{2}, 2 \vartheta_{01}(0)^{4}, \\
& -2 \vartheta_{00}(0)^{2} \vartheta_{10}(0)^{2}, 2 \vartheta_{01}(0)^{2} \vartheta_{10}(0)^{2},-2 \vartheta_{10}(0)^{4},-2 \vartheta_{01}(0)^{2} \vartheta_{10}(0)^{2}, \\
& \vartheta_{10}(0)^{4}, 2 \vartheta_{01}(0)^{2} \vartheta_{10}(0)^{2}, \vartheta_{01}(0)^{4},-2 \vartheta_{00}(0)^{2} \vartheta_{10}(0)^{2},-2 \vartheta_{00}(0)^{2} \vartheta_{01}(0)^{2}, \\
& \left.-4 \vartheta_{10}(0)^{4}, 4 \vartheta_{10}(0)^{4}, \vartheta_{00}(0)^{4},-2 \vartheta_{00}(0)^{2} \vartheta_{10}(0)^{2}, \vartheta_{10}(0)^{4}\right) .
\end{aligned}
$$

In the following, we fix the identification $M=\boldsymbol{P}^{23}$.

\section{§3. Application to the Moduli Theory}

In this section where the author owes to [8] to a great extent, we consider the totality of the Chow point $c\left(\phi\left(E_{\tau}\right)\right)(\tau \in H)$.

3.1. We start with recalling some facts from the theory of elliptic modular functions.

The homogeneous modular group $\operatorname{SL}(2, Z)$ acts on $H$ by

$$
\left(\begin{array}{ll}
a & b \\
c & d
\end{array}\right) \tau=\frac{a \tau+b}{c \tau+d}
$$

For any positive integer $N$, the principal congruence subgroup $\Gamma(N)$ of level $N$ of $\operatorname{SL}(2, Z)$ is, by definition

$$
\Gamma(N)=\operatorname{Ker}(\operatorname{SL}(2, Z) \stackrel{\bmod N}{\longrightarrow} \operatorname{SL}(2, Z / N \boldsymbol{Z})) .
$$

By a modular form of weight $k$ ( $k$ a positive integer) and level $N$, we mean a holomorphic function $f(\tau)$ on $H$ such that 


$$
\forall \gamma=\left(\begin{array}{ll}
a & b \\
c & d
\end{array}\right) \in \Gamma(N) ; \quad f(\gamma \tau)=(c \tau+d)^{k} f(\tau)
$$

and that $f(\tau)$ is bounded at $Q \cup\{\infty\}$ (for further details, [8]).

We are concerned with $\Gamma(4)$.

The inequivalent cusps of $\Gamma(4)$ are $\infty, 0,1 / 2,1,2$ and 3 .

We denote the extended upper half plane by $\tilde{H}$, i.e.,

$$
\tilde{H}:=H \cup \boldsymbol{Q} \cup\{\infty\} .
$$

The compactification $\widetilde{H / \Gamma(4)}$ of $H / \Gamma(4)$ is set-theoretically

$$
\widetilde{H / \Gamma(4)}=\tilde{H} / \Gamma(4)=H / \Gamma(4) \cup\left\{\infty, 0, \frac{1}{2}, 1,2,3\right\}
$$

The followings are known ([8]):

(i). $\vartheta_{i j}(\tau \mid 0)^{2}$ are modular forms on the upper half plane $H$ of weight 1 of level 4 ,

(ii). The map $\theta^{\prime}: H \rightarrow \boldsymbol{P}^{2}$ defined by

$$
\tau \mapsto \theta^{\prime}(\tau)=\left(\vartheta_{00}(\tau \mid 0)^{2}, \vartheta_{01}(\tau \mid 0)^{2}, \vartheta_{10}(\tau \mid 0)^{2}\right)
$$

induces a holomorphic embedding $\theta: H / \Gamma(4) \rightarrow \boldsymbol{P}^{2}$.

Let $\left(y_{0}, y_{1}, y_{2}\right)$ be the homogeneous coordinates in $\boldsymbol{P}^{2}$ corresponding to the above map, and let $C_{0}$ be a plane conic defined by

$$
y_{0}^{2}-y_{1}^{2}-y_{2}^{2}=0 .
$$

We define an open set $C_{0}^{\prime}$ of $C_{0}$ by

$$
C_{0}^{\prime}:=C_{0}-\{(1,0, \pm 1),(1, \pm 1,0),(0,1, \pm i)\}
$$

Then, $\operatorname{Im}(\theta)=C_{0}^{\prime}$.

(iii). The holomorphic map $\theta$ is extended to the compactification $\widetilde{H / \Gamma(4)}$ so that the following diagram is commutative:

$$
\begin{aligned}
& \widetilde{H / \Gamma(4)} \stackrel{\sim}{\longrightarrow} C_{0} \\
& \cup U \text {. } \\
& H / \Gamma(4) \stackrel{\sim}{\longrightarrow} C_{0}^{\prime}
\end{aligned}
$$

We also denote by $\theta$ the extended holomorphic map.

Let $p$ be any one of the inequivalent cusps $\infty, 0,1 / 2,1,2$ and 3 . Then $\theta(p)$ is given by the following table: 


\begin{tabular}{|c|c|c|c|c|c|c|}
\hline$p$ & $\infty$ & 0 & $1 / 2$ & 1 & 2 & 3 \\
\hline$\theta(p)$ & $(1,1,0)$ & $(1,0,1)$ & $(1,-1,0)$ & $(0,1, i)$ & $(1,0,-1)$ & $(0,1,-i)$ \\
\hline
\end{tabular}

3.1.1. For every $\tau \in H$, we denote by $c(\tau)$ the Chow-point $c\left(\phi\left(E_{\tau}\right)\right)$ of the projective elliptic normal curve $\phi\left(E_{\tau}\right)$. By 2.3, $c(\tau) \in M=\boldsymbol{P}^{23}$ and $c(\tau)$ is obtained from replacing $\vartheta_{i j}(0)$ by $\vartheta_{i j}(\tau \mid 0)$ in (6). Therefore, a system of homogeneous coordinates of $c(\tau)$ is given by modular forms of weight 2 and of level 4 .

We are interested in the subset $\{c(\tau) \mid \tau \in H\}$ of $M$. Let $C$ be the closure of the set $\{c(\tau) \mid \tau \in H\}$ in the Zariski topology.

We define a holomorphic map $c: H \rightarrow C$ by $\tau \mapsto c(\tau)$.

THEOREM 2. $C$ is a non-singular rational curve of degree 4 in $M$ having the following properties:

(1) $\{c(\tau) \mid \tau \in H\} \subset C$ is a Zariski open subset.

(2) $\exists$ an isomorphism: $C_{0} \stackrel{\sim}{\longrightarrow} C$ such that the diagram:

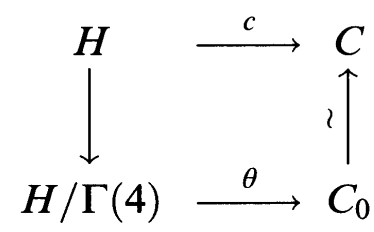

is commutative.

Proof. Let $C_{0}$ be the plane conic defined in (ii) of 3.1. By $C^{\prime}$, we mean the image of the 2-uple embedding, which is denoted by $\rho$, of $C_{0} . C^{\prime}$ is a non-singular rational curve of degree 4 in $\boldsymbol{P}^{5}$ (which lies on a hyperplane of $\boldsymbol{P}^{5}$ ).

Then, $\forall \tau \in H$;

$$
\begin{aligned}
\rho\left(\theta^{\prime}(\tau)\right)= & \left(\vartheta_{00}(\tau \mid 0)^{4}, \vartheta_{00}(\tau \mid 0)^{2} \vartheta_{01}(\tau \mid 0)^{2}, \vartheta_{00}(\tau \mid 0)^{2} \vartheta_{10}(\tau \mid 0)^{2}, \vartheta_{01}(\tau \mid 0)^{4}\right. \\
& \left.\vartheta_{01}(\tau \mid 0)^{2} \vartheta_{10}(\tau \mid 0)^{2}, \vartheta_{10}(\tau \mid 0)^{4}\right) .
\end{aligned}
$$

While, by (6),

$\exists \pi^{\prime}: M \rightarrow P^{4}$ a composition of a linear projection and a (trivial) projective transformation such that $\forall \tau \in H$;

$$
\begin{aligned}
\pi^{\prime}(c(\tau))= & \left(\vartheta_{00}(\tau \mid 0)^{4}, \vartheta_{00}(\tau \mid 0)^{2} \vartheta_{01}(\tau \mid 0)^{2}, \vartheta_{00}(\tau \mid 0)^{2} \vartheta_{10}(\tau \mid 0)^{2}, \vartheta_{01}(\tau \mid 0)^{4}\right. \\
& \left.\vartheta_{01}(\tau \mid 0)^{2} \vartheta_{10}(\tau \mid 0)^{2}, \vartheta_{10}(\tau \mid 0)^{4}\right) \\
= & \rho\left(\theta^{\prime}(\tau)\right) .
\end{aligned}
$$

$\pi^{\prime}$ is not unique, and so we fix such an $\pi^{\prime}$. 
Conversely, $\rho\left(\theta^{\prime}(\tau)\right)$ determines $c(\tau)$. Hence, $\pi^{\prime}$ induces a bijection

$$
\{c(\tau) \mid \tau \in H\} \stackrel{\sim}{\longrightarrow}\left\{\rho\left(\theta^{\prime}(\tau)\right) \mid \tau \in H\right\}=\rho\left(C_{0}^{\prime}\right) .
$$

Since $\rho\left(C_{0}^{\prime}\right)$ is a Zariski open set of $C^{\prime}, C$ becomes an algebraic curve which is isomorphic to $C_{0}$, using the bijection.

Q.E.D.

This theorem shows that for any $\tau \in H$, the Chow point $c(\tau)$ of the projective elliptic normal curve $\phi\left(E_{\tau}\right)$ is essentially given by the image of the 2uple embedding of the point $\left(\vartheta_{00}(\tau \mid 0)^{2}, \vartheta_{01}(\tau \mid 0)^{2}, \vartheta_{10}(\tau \mid 0)^{2}\right)$.

In our case, the appearance of the 2-uple embedding is primarily based on the fact that elliptic normal curves of degree 4 in $\boldsymbol{P}^{3}$ are complete intersections by quadratic forms.

It seems to the author that: when we consider elliptic normal curves of degree $r>4$ embedded in $P^{r-1}$ by using theta functions, the level of the corresponding principal congruence subgroup will be biggeer than 4 . Therefore if $r$ is sufficiently large, the genus of the corresponding curve $C$ is bigger than 0 . Moreover the genus of $C$ will be bigger as long as $r$ is larger.

3.1.2. Next we consider the compactification of $H / \Gamma(4)$.

By the abuse of notations, we denote by $\rho$ the isomorphism $C_{0} \stackrel{\sim}{\longrightarrow} C$ in the Th. 2 .

When $p$ is any one of the inequivalent cusps, let $X_{p}$ be the specialization of the projective curve $X_{\tau}(\tau \in H)$ over the specialization $p$ of $\tau$. The equations for $X_{p}$ are given by the following table ([8], p. 57):

\begin{tabular}{|c|c|c|c|c|c|c}
\hline$p$ & $\infty$ & 0 & $1 / 2$ & 1 & 2 & 3 \\
\hline \multirow{2}{*}{ equations } & $x_{0}^{2}=x_{1}^{2}$ & $x_{0}^{2}=x_{2}^{2}$ & $x_{0}^{2}=-x_{1}^{2}$ & $x_{0}^{2}=-i x_{3}^{2}$ & $x_{0}^{2}=-x_{2}^{2}$ & $x_{0}^{2}=i x_{3}^{2}$ \\
& $x_{2}^{2}=-x_{3}^{2}$ & $x_{1}^{2}=x_{3}^{2}$ & $x_{2}^{2}=x_{3}^{2}$ & $x_{1}^{2}=-i x_{2}^{2}$ & $x_{1}^{2}=-x_{3}^{2}$ & $x_{1}^{2}=i x_{2}^{2}$ \\
\hline
\end{tabular}

Thus, for any $p, X_{p}$ is a 1-cycle of degree 4 , consisting of 4 lines.

Since Chow-forms are compatible with specializations, the six Chow-forms $P_{X_{\infty}}, P_{X_{0}}, P_{X_{1 / 2}}, P_{X_{1}}, P_{X_{2}}$ and $P_{X_{3}}$ are obtained by specializations.

Let $c(p)$ be the Chow-point $c\left(X_{p}\right)$ of the cycle $X_{p}$. Then, $(c(p), \theta(p))$ is a specialization of $(c(\tau), \theta(\tau))$. Therefore $c(p)$ lies on the curve $C$.

Let $\Gamma(4)(p)$ be the orbit of $p$ under $\Gamma(4)$. Then

$$
Q \cup\{\infty\}=\Gamma(4)(\infty) \cup \Gamma(4)(0) \cup \Gamma(4)\left(\frac{1}{2}\right) \cup \Gamma(4)(1) \cup \Gamma(4)(2) \cup \Gamma(4)(3)
$$


Now, we define a map

$$
c: \tilde{H} \rightarrow C
$$

by

$$
c(\tau):=\left\{\begin{array}{ll}
c(\tau), & \text { if } \tau \in H \\
c(p), & \text { if } \tau \in \Gamma(4)(p)
\end{array} .\right.
$$

We note that for any $p^{\prime} \in \Gamma(4)(p)$, the limiting cycle $X_{p^{\prime}}$ of $X_{\tau}$, as $\tau \rightarrow p^{\prime}$, is uniquely determined and then $X_{p^{\prime}}=X_{p}$.

Summarizing we have

COROLlary 1. The diagram:

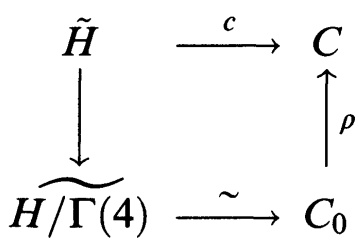

is commutative.

Now, we recall the topology on $\tilde{H}$. A fundamental system of neighbourhoods $\mathfrak{B}(x)$ of $x(x \in \tilde{H})$ is defined by

$\mathfrak{B}(x):=\left\{\begin{array}{ll}\text { the usual one, } & \text { if } x \in H \\ \text { the set of all }\{p, \text { the interior of a circle in } & \\ H \text { tangent to the real axis at } p\}, & \text { if } \infty \neq x=p \notin H \\ \text { the set of all }\{\infty, \tau \mid \tau \in H, \operatorname{Im}(\tau)>c\}(c>0), & \text { if } x=\infty\end{array}\right.$.

The topological space $\widetilde{H / \Gamma(4)}=\tilde{H} / \Gamma(4)$ is defined to be the quotient space of $\tilde{H}$. Hence, $c$ becomes a continuous map.

3.2. Finally, we consider the Chow variety $\mathscr{C}$ of the 1-cycles of degree 4 in $\boldsymbol{P}^{3} . \mathscr{C}$ is an algebraic set in the 104-dimensional projective space $\boldsymbol{P}\left(\Gamma_{4}\right)$. Let $\boldsymbol{\Sigma}$ be the component of $\mathscr{C}$ whose general point corresponds to an irreducible curve which is a complete intersections of two quadrics. $\Sigma$ is of dimension 16 ([1]).

$\Sigma$ is stable under the canonical action of $P G L(4)$.

Proof. Let $c \in \Sigma$ be any point and let $X$ be the 1-cycle of degree 4 in $\boldsymbol{P}^{3}$ corresponding to $c$. 
If $X$ is an irreducible curve, then $\forall \gamma \in P G L(4), X^{\gamma}$ is also irreducible.

Hence, $c^{\gamma}=c\left(X^{\gamma}\right) \in \Sigma$.

Next, we assume that $X$ is not irreducible. Then there is an irreducible curve $X_{0}$ s.t. $c_{0}=c\left(X_{0}\right) \in \Sigma$ and that $c$ is a specialization of $c_{0}$.

Then $c^{\gamma}$ is also a specialization of $c_{0}^{\gamma}$.

Since $c_{0}^{\gamma} \in \Sigma$, we have $c^{\gamma} \in \Sigma$.

Q.E.D.

As to the structure of $\Sigma$, we have the following:

Corollary 2. Let $C$ be the rational curve as in Th. 2. Then there is a natural dominating morphism:

$$
\mathrm{SL}(4, C) \times C \rightarrow \Sigma
$$

induced by the canonical action.

Proof. By Th. 2, $C(\subset M)$ lies on $\Sigma$. Let $\sigma: \operatorname{SL}(4, C) \times \Sigma \rightarrow \Sigma$ be the canonical action.

Let $f: \operatorname{SL}(4, C) \times C \rightarrow \Sigma$ be the morphism induced by $\sigma,(\gamma, c) \mapsto c^{\gamma}$.

Let $\rho: C_{0} \stackrel{\sim}{\longrightarrow} C$ be the isomorphism.

$C_{0}^{\prime}=C_{0}-\{6$ cusps $\}$ is the open set of $C_{0} . \rho\left(C_{0}^{\prime}\right)$ is an open set of $C$.

We denote by $f^{\prime}$ the restriction of $f$ to $\operatorname{SL}(4, C) \times \rho\left(C_{0}^{\prime}\right)$.

Now, for any $\tau \in H$, the projective curve $X_{\tau}=\phi\left(E_{\tau}\right)$ is a non-singular curve embedded by a complete linear system of degree 4 . Hence $X_{\tau}$ is Chow-stable (Th. 4.15 in [7]). In other words, the orbit of the Chow point $c\left(X_{\tau}\right)$ by $\operatorname{SL}(4, C)$ is closed and the stabilizer is finite.

$$
\therefore \operatorname{dim} \operatorname{Im} f^{\prime}=\operatorname{dim} \operatorname{SL}(4, C)+\operatorname{dim} \rho\left(C_{0}^{\prime}\right)=16=\operatorname{dim} \Sigma .
$$

Hence, $f$ dominates $\Sigma$.

Q.E.D.

\section{References}

[1] D. Eisenbud-J. Harris, The dimension of the Chow variety of curves, Comp. Math., 83, 1992, 291-310.

[2 ] W. V. D. Hodge-Pedoe, "Methods of Algeraic Geometry", Vol. 2, Cambridge University Press (1968).

[3] A. Hurwitz, Über die Trägheitsformen eines algebraischen Moduls, Annali die Mathematica. pura ed applics, serie III, t. 20, 1913, 113-151.

[4] J. Igusa, On the arithmetic normality of the grassmann variety, Proc. N.A.S., Vol. 40 (1954), pp. 309-313

[5] J. Igusa, "Theta functions", Grundlehren der mathematischen Wissenschaften, Bd. 194, Springer-Verlag, Berlin Heidelberg New-York, 1972. 
[6] A. Krazer, "Lehrbuch der Thetafunktionen", Chelsea, New York, 1970.

[7] D. Mumford, Stability of projective varieties. L'Ens. Math., 24, (1977).

[ 8 ] D. Mumford, "Tata Lectures on Theta I", Progress in Math., 28, Birkhauser (1983)

[9] T. Ono, "Variations on a Theme of Euler", Jikkyo Publishing, Tokyo, 1980 (in Japanese); Plenum Publishing, New York, 1994 (English translation)

[10] B. L. van der Waerden, "Einführung in die algebraische Geometrie", Springer-Verlag, Berlin Heidelberg New-York, 1973.

Tatsuji TANAKA

Department of Mathematics

Faculty of Science and Engineering

Saga University

Saga 840-8502, Japan

e-mail: tanaka@ms.saga-u.ac.jp 